\title{
Accurate 2-D AOA Estimation and Ambiguity Resolution for a Single Source under Fixed Uniform Circular Arrays
}

\author{
Le Zuo and Jin Pan \\ Department of Microwave Engineering, University of Electronic Science and Technology of China, Chengdu 611731, China \\ Correspondence should be addressed to Le Zuo; zorro1204@163.com
}

Received 25 July 2017; Revised 7 October 2017; Accepted 22 October 2017; Published 12 December 2017

Academic Editor: Yuan Yao

Copyright ( 2017 Le Zuo and Jin Pan. This is an open access article distributed under the Creative Commons Attribution License, which permits unrestricted use, distribution, and reproduction in any medium, provided the original work is properly cited.

\begin{abstract}
This paper presents an analytic algorithm for accurate two-dimensional (2-D) angle of arrival (AOA) estimation of a single source under fixed uniform circular arrays (UCAs). Algebraic and explicit formulations for 2-D AOA estimation are first developed in the Fourier domain. It is shown that three is the minimum number of antennas for 2-D AOA estimation based on phase measurement. Then a signal model for phase extraction is established with equivalent phase noises through observations of signal samples corrupted by additive Gaussian white noise. Under fixed UCAs, 2-D AOA estimation of a single source would suffer from phase ambiguity, and hence, ambiguity resolution is also addressed in the Fourier domain by integer search. Numerical examples are provided to verify the effectiveness and appealing performance of the proposed 2-D AOA estimation algorithm.
\end{abstract}

\section{Introduction}

Fast and accurate estimation of the two-dimensional (2-D) angle of arrival (AOA) of incident plane waves is important in array signal processing due to its applications in radar, sonar, and mobile communications. Uniform circular array (UCA) is extensively utilized in the context of 2-D AOA estimation due to its attractive advantages, including $360^{\circ}$ azimuthal coverage, almost unchanged directional pattern, and additional elevation angle information $[1,2]$. Recently, a lot of literatures have been reported on the AOA estimation with UCAs. In [3], spatial averaging algorithm and spectrum search were applied to 2-D estimation with UCAs. Reference [4] developed two eigenstructure-based algorithms for 2-D estimation with UCAs. However, these methods introduce errors in the form of bias and excess variance, and hence, the obtained estimates may be far from optimal [5]. Furthermore, these methods involve eigenvalue decomposition; thus, the computational load is significant. Liao et al. proposed a generalized algorithm for 2-D AOA estimation based on the least square estimation [2]. As will be shown in Section 2 , if the antenna element number is even, our algorithm is equivalent to the generalized method. However, if the antenna element number is odd, the simulated accuracy by the generalized method is lower than our algorithm, as will be shown in Section 4.

In addition, it is well known that high AOA estimation accuracy can be obtained from large apertures. However, the measurement of phase difference can only be made modulo of $2 \pi$, which leads to an ambiguity in determining the AOA of the source [6]. To solve the phase ambiguity, a modulo conversion method [7] was proposed, but it is inherently developed for linear array interferometers and cannot be directly applied to UCAs, for UCA's phase differences are dependent on both elevation and azimuth angles. As regards ambiguity resolution under UCAs, rotary ways were used [8-10], whereas rotary interferometers face the problem of source correspondence and real-time applications. In [11], a method called subarray grouping and ambiguity searching was proposed and the rough angle estimation was achieved by searching the nearest value among subarrays. However, the antenna elements were in pairs, and hence, the number of antenna elements must be even.

To avoid eigenvalue calculation, in this paper, we propose an analytical 2-D AOA estimation algorithm under fixed UCAs. The algorithm is based on the Fourier analysis of the phase around the circular aperture. The underlying 
AOA estimation problem is reformulated as expansion coefficient calculation problem. The solutions to 2-D AOAs are explicit discrete Fourier transform (DFT) of antenna outputs that sample the phases around the circular aperture. A signal model for phase extraction is then established with equivalent phase noises through observations of signal samples corrupted by additive white Gaussian noise (AWGN). Furthermore, without rotation, we address ambiguity resolution by finding the missing spectrum of ambiguity numbers through integer search. Numerical examples show the effectiveness and appealing performance of the proposed algorithm.

This paper contributes to the area of 2-D AOA estimation in the following aspects:

(1) Algebraic formulations for accurate 2-D AOA estimation under UCAs are presented with low computational complexity.

(2) The estimation algorithm sufficiently exploits the centrosymmetry and periodicity of the circular aperture by Fourier transform, resulting in an algebraic solution to 2-D AOAs.

(3) A novel ambiguity resolution based on integer search and inverse Fourier transform is developed for fixed UCAs, and hence, it is applicable to real-time AOA estimations.

The rest of this paper is organized as follows. In Section 2, phase-based expressions for continuous and discrete phases around a circular aperture are first developed, which decouples the 2-D AOA parameters by Fourier transform, and then a signal model is established for phase extraction in AWGN. Section 3 addresses ambiguity resolution based on DFT and integer search. Numerical simulations are presented in Section 4. Section 5 concludes this paper.

\section{AOA Estimation Algorithm}

In this section, Fourier transform is first applied to the noiseless periodic phase distribution around a continuous circular aperture and then to the noiseless discrete phase samples. In order to extract the phases for the AOA estimation, a signal model is established, in which the phase noises are equivalent to the AWGN in the time domain. Moreover, the proposed algorithm is compared to a previous method [2].

2.1. Continuous Aperture. Consider a circular aperture located at $\left(\rho_{0}, \pi / 2, \varphi\right)$ in the spherical coordinate system of $(\rho, \theta, \varphi)$, as shown in Figure 1. The phase of the electric field of an incident wave from $\theta_{s}$ to $\varphi_{s}$ can be written as

$$
\Phi(\varphi)=\Phi\left(\rho_{0}, \frac{\pi}{2}, \varphi\right)=k \rho_{0} \sin \theta_{s} \cos \left(\varphi_{s}-\varphi\right)+\Phi_{0}
$$

where the azimuth angle $\varphi_{s} \in[0,2 \pi)$ is measured counterclockwise from the $x$-axis and the elevation angle $\theta_{s} \in[0, \pi)$ is measured down from the $z$-axis, $k=2 \pi / \lambda$ is the wave number in free space, and $\lambda$ is the wavelength. The first term depends on the element position and contains the unknown

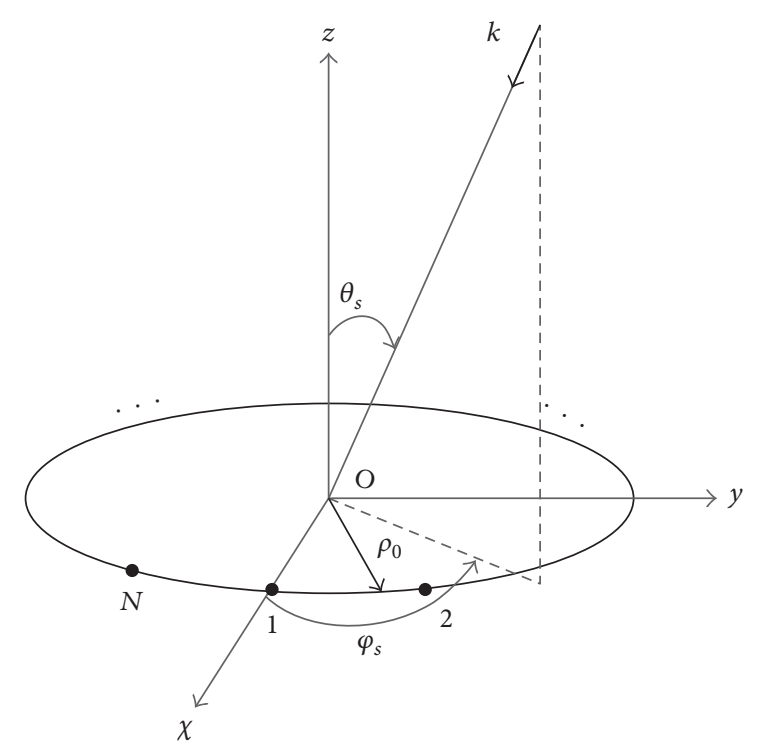

Figure 1: Circular aperture geometry with a single incident wave.

AOA parameters. The second term, that is, $\Phi_{0}$, is a constant and represents the initial phase of the incident wave, which can be interpreted as the phase of the incident wave arriving at the center of the array.

Noticing that the phase $\Phi(\varphi)$ is a periodic function of $\varphi$, we apply Fourier transform to (1) and obtain

$$
\begin{aligned}
\Psi(n)= & \int_{0}^{2 \pi} \Phi(\varphi) \exp (j n \varphi) d \varphi \\
= & \pi k \rho_{0} \sin \theta_{s}\left[\delta(n-1) \exp \left(j \varphi_{s}\right)+\delta(n+1) \exp \left(-j \varphi_{s}\right)\right] \\
& +2 \pi \Phi_{0} \delta(n),
\end{aligned}
$$

where

$$
\delta(n)= \begin{cases}1, & n=0 \\ 0, & n \neq 0\end{cases}
$$

Hence, we get the dependence of 2-D AOA on $\Psi(1)$, namely,

$$
\Psi(1)=\pi k \rho_{0} \sin \theta_{s} \exp \left(j \varphi_{s}\right) .
$$

It is obvious that the two angular parameters are decoupled in (4), where the elevation dependence $\theta_{s}$ is on the magnitude of $\Psi(1)$ and azimuth dependence $\varphi_{s}$ is on the phase of $\Psi(1)$, that is,

$$
\begin{aligned}
& \theta_{s}=\sin ^{-1}\left(\frac{1}{\left(\pi k \rho_{0}\right)} \cdot|\Psi(1)|\right), \\
& \varphi_{s}=\arg (\Psi(1)) .
\end{aligned}
$$

Equations (5) and (6) imply that the 2-D AOAs are decoupled and obtained by Fourier transform, given the continuous phase distribution around a circular aperture. 
2.2. Discrete Phase Samples. A UCA, as shown in Figure 1, can be deemed as $N$ equally placed identical antennas that sample the fields of the circular aperture, that is, the noiseless phases of the output of the antenna elements are denoted by

$$
\Phi_{s}(\varphi)=\Phi(\varphi) \sum_{i=1}^{N} \delta\left(\varphi-\varphi_{i}\right)=\sum_{i=1}^{N} \Phi\left(\varphi_{i}\right) \delta\left(\varphi-\varphi_{i}\right)
$$

where $\varphi_{i}=(i-1) \Delta \varphi$ and $\Delta \varphi=2 \pi / N$. Let us define $\Phi_{i}$ as the phase samples at $\varphi_{i}$, for example, $\Phi\left(\varphi_{i}\right)=\Phi_{i}$, and according to (1), we have

$$
\Phi_{i}=k \rho_{0} \sin \theta_{s} \cos \left(\varphi_{s}-\varphi_{i}\right)+\Phi_{0}
$$

Similarly, we apply Fourier transform to the second equality in (7), and by changing the order of integral and summation, we get

$$
\begin{aligned}
\Psi_{s}(n) & =\int_{0}^{2 \pi} \Phi_{s}(\varphi) \exp (j n \varphi) d \varphi \\
& =\int_{0}^{2 \pi} \sum_{i=1}^{N} \Phi_{i} \delta\left(\varphi-\varphi_{i}\right) \exp (j n \varphi) d \varphi=\Delta \varphi \sum_{i=1}^{N} \Phi_{i} \exp \left(j n \varphi_{i}\right) .
\end{aligned}
$$

From the convolution theorem of Fourier transform, we also get from the first equality of (7)

$$
\begin{aligned}
\Psi_{s}(n) & =\int_{0}^{2 \pi} \Phi_{s} \exp (j n \varphi) d \varphi \\
& =\int_{0}^{2 \pi} \Phi(\varphi) \exp (j n \varphi) d \varphi * \int_{0}^{2 \pi} \sum_{i=1}^{N} \delta\left(\varphi-\varphi_{i}\right) \exp (j n \varphi) d \varphi \\
& =\Psi(n) * \sum_{l=-\infty}^{+\infty} \delta(n-l N)=\sum_{l=-\infty}^{+\infty} \Psi(n-l N),
\end{aligned}
$$

where $\left({ }^{*}\right)$ denotes the convolution operator. Careful examination of (2) shows $\Psi(n)$ is nonzero only when $n=-1,0,1$. Therefore, when $N \geq 3$, the value of $\Psi_{s}(1)$ remains the same as (4). Hence, by combining (9) and (4), we get

$$
\Psi_{s}(1)=\Delta \varphi \sum_{i=1}^{N} \Phi_{i} \exp \left(j \varphi_{i}\right)=\pi k \rho_{0} \sin \theta_{s} \exp \left(j \varphi_{s}\right),
$$

which is the discrete Fourier transform (DFT) of $\Phi_{i}$. It is worth noting that the minimum number for 2-D AOA estimation of a single source with a UCA is three.

Finally, considering (5) and (6), we get the analytic and explicit formulations of the 2-D AOAs, namely,

$$
\begin{aligned}
& \theta_{s}=\sin ^{-1}\left(\frac{2}{\left(N k \rho_{0}\right)} \cdot\left|\sum_{i=1}^{N} \Phi_{i} \exp \left(j \varphi_{i}\right)\right|\right), \\
& \varphi_{s}=\arg \left(\sum_{i=1}^{N} \Phi_{i} \exp \left(j \varphi_{i}\right)\right) .
\end{aligned}
$$

Noteworthily, the initial phase $\Phi_{0}$ is immaterial, provided that it is not shown in the estimation formulations and it is the same for all elements. Alternatively and conveniently, the phases can be measured with respect to a particular antenna.

2.3. Phase Extraction. As indicated in (12) and (13), given the discrete sampled phases around a circular aperture, the 2-D AOAs can be obtained without accuracy loss. This subsection examines the extraction of the receiver's phases from signal samples corrupted by AWGN.

Consider a UCA with $N$ identical elements illuminated by a single far-field source. The $t$ th sample from the $i$ th digital receiver is assumed to have the form

$$
x_{i}(t)=A e^{j \omega t+j \Phi_{i}}+n_{i}(t)
$$

where $A$ is the magnitude of the signal and $\varepsilon_{i}(t)$ is an AWGN with zero mean and covariance $\sigma_{n}^{2}$. The noises of each receiver are independent. The signal-to-noise ratio (SNR) is defined by $\mathrm{SNR}=\sigma_{s}^{2} / A^{2}$.

The unambiguous phase is considered here, and the phase ambiguity is delayed to Section 3. At moderately high SNR, the AWGN can be converted into an equivalent additive phase noise [12], that is,

$$
\tilde{\Phi}_{i}=\arg \left(x_{i}\right)=\Phi_{i}+\varepsilon_{i}
$$

where $\varepsilon_{i}$ is the phase noise of the $i$ th receiver. The frequency of the signal is assumed to be accurately estimated using a number of well-known techniques [13]. The phase of each receiver output can be obtained by [14]

$$
\tilde{\Phi}_{i}=\arg \left(\frac{1}{M} \sum_{t=1}^{M} x_{i}(t) e^{-j \omega T t}\right)
$$

where $T$ is the inverse of the constant sampling rate. The variance of $\varepsilon_{i}$ is given by

$$
\operatorname{var}\left(\varepsilon_{i}\right)=\frac{1}{(2 \cdot M \cdot \mathrm{SNR})},
$$

where $M$ is the number of snapshots.

2.4. Equivalence to Previous Method [2]. Interestingly, if the element number is even, in the least square estimation $\widehat{b}=\left(\mathbf{A}^{T} \mathbf{A}\right)^{-1} \mathbf{A}^{T} \mathbf{w}$, that is, (11) of [2], $\left(\mathbf{A}^{T} \mathbf{A}\right)^{-1}$ becomes

$$
\begin{aligned}
\left(\mathbf{A}^{T} \mathbf{A}\right)^{-1} & =\left[\begin{array}{cc}
\sum_{i=1}^{N / 2} \cos ^{2} \varphi_{i}, & \sum_{i=1}^{N / 2} \cos \varphi_{i} \sin \varphi_{i} \\
\sum_{i=1}^{N / 2} \cos \varphi_{i} \sin \varphi_{i}, & \sum_{i=1}^{N / 2} \sin ^{2} \varphi_{i}
\end{array}\right]^{-1} \\
& =\frac{4}{N}\left[\begin{array}{cc}
1, & 0 \\
0, & 1
\end{array}\right]
\end{aligned}
$$


and because the element number is even, $\cos \varphi_{i}=-\cos \varphi_{i+N / 2}$ and $\sin \varphi_{i}=-\sin \varphi_{i+N / 2}$, and $\mathbf{A}^{T} \mathbf{w}$ becomes

$$
\begin{aligned}
\mathbf{A}^{T} \mathbf{w} & =\left[\sum_{i=1}^{\frac{N}{2}}\left(\Phi_{i}-\Phi_{i+\frac{N}{2}}\right) \cos \varphi_{i}, \sum_{i=1}^{\frac{N}{2}}\left(\Phi_{i}-\Phi_{i+\frac{N}{2}}\right) \sin \varphi_{i}\right]^{T} \\
& =\left[\sum_{i=1}^{N} \Phi_{i} \cos \varphi_{i}, \sum_{i=1}^{N} \Phi_{i} \sin \varphi_{i}\right]^{T} .
\end{aligned}
$$

By letting $\widehat{b}_{1}+j \widehat{b}_{2}=\Psi_{s}(1) /(2 \pi)$, we get that (12) and (13) are equivalent to (12) in [2].

Moreover, when the number of antennas is odd, the least square estimation in [2] can be reformulated by $N$ terms instead of $(N-l)$ terms, where $l$ denotes $n$ in [2], that is, (14a) in [2] which can be rewritten as

$$
\begin{aligned}
& \overline{\mathbf{w}}=\left[\begin{array}{llllll}
w_{1,1+l} & w_{2,2+l} & \cdots & w_{N-l, N} & \cdots & w_{N, l}
\end{array}\right]^{T}, \\
& \overline{\mathbf{A}}=\left[\begin{array}{cc}
\cos \varphi_{1}-\cos \varphi_{1+l} & \sin \varphi_{1}-\sin \varphi_{1+l} \\
\cos \varphi_{2}-\cos \varphi_{2+l} & \sin \varphi_{2}-\sin \varphi_{2+l} \\
\vdots & \vdots \\
\cos \varphi_{N-l}-\cos \varphi_{N} & \sin \varphi_{N-l}-\sin \varphi_{N} \\
\vdots & \vdots \\
\cos \varphi_{N}-\cos \varphi_{l} & \sin \varphi_{N}-\sin \varphi_{l}
\end{array}\right]
\end{aligned}
$$

Then (17) in [2] is the same as (12) and (13). It is worth noting that if the number of terms in the least square estimation is equal to that of antennas, the solutions to the least square are the same and are irrelevant to the choice of $l$.

\section{Ambiguity Resolution}

It is well known that high AOA estimation accuracy can be obtained for large apertures. However, when $\rho_{0}>\lambda / 2$, the phase range may exceed $2 \pi$, while the measurement of phase difference, that is, (16), can only be made modulo $2 \pi$, which leads to an ambiguity in determining the direction of the incident wave. The angle estimation exploited the centrosymmetry and periodicity of the circular aperture, and in this section, we continue to adopt the particular properties of the circular aperture and Fourier transform for ambiguity resolution.

The phases measured at each antenna can be written as

$$
\tilde{\Phi}_{i}=\tilde{\Phi}_{i}^{\prime}-2 \pi m_{i}, \quad \tilde{\Phi}_{i}^{\prime} \in(-\pi, \pi],
$$

where $m_{i}$ is an integer and recognized as the ambiguous number. Because $\Phi_{0}$ is irrelevant to the solution of 2-D AOAs, we let $\Phi_{0}$ be equal 0 . Substitution of (8) into (22) yields

$$
k \rho \sin \theta_{s} \cos \left(\varphi_{s}-\varphi_{i}\right)=\Phi_{i}^{\prime}-2 \pi m_{i}, \quad i=1,2, \ldots, N .
$$

Discrete Fourier transform of both sides gives

$$
\begin{aligned}
\Psi(n) & =\sum_{i=1}^{N} k \rho \sin \theta_{s} \cos \left(\varphi_{s}-\varphi_{i}\right) e^{j n \varphi_{i}}=\sum_{i=1}^{N} \Phi_{i}^{\prime} e^{j n \varphi_{i}}-2 \pi \sum_{i=1}^{N} n_{i} e^{j n \varphi_{i}} \\
& =\Psi^{\prime}(n)-2 \pi \Psi_{A N}(n), \quad n=-\left\lfloor\frac{N}{2}\right\rfloor, \ldots,\left\lfloor\frac{N}{2}\right\rfloor-1,
\end{aligned}
$$

where $\lfloor x\rfloor$ denotes the integer part of $x$ and

$$
\begin{aligned}
\Psi^{\prime}(n) & =\sum_{i=1}^{N} \Phi_{i}^{\prime} e^{-j n \varphi_{i}}, \\
\Psi_{A N}(n) & =\sum_{i=1}^{N} m_{i} e^{-j n \varphi_{i}}
\end{aligned}
$$

are the DFTs of ambiguous phases and ambiguity numbers, respectively. Equation (25) implies that $m_{i}$ is the inverse discrete Fourier transform of $\Psi_{A N}(n)$. Noticing $\Psi(n)$ is nonzero only when $n= \pm 1$, and comparing the coefficients of DFT, we have

$$
\begin{aligned}
& n=1, \Psi_{A N}(1)=\frac{1}{2 \pi}\left(\Psi^{\prime}(1)-\Psi(1)\right), \\
& n=-1, \Psi_{A N}(-1)=\frac{1}{2 \pi}\left(\Psi^{\prime}(-1)-\Psi(-1)\right), \\
& n=\text { others, } \Psi_{A N}(n)=\frac{1}{2 \pi} \Psi^{\prime}(n) .
\end{aligned}
$$

Provided that $\Psi^{\prime}(n)$ is obtained from the phase measurement, $\Psi(1)$ and $\Psi(-1)$ are unknown, and $\Psi_{A N}(1)$ and $\Psi_{A N}(-1)$ are to be determined. After $\Psi_{A N}(n)$ is all determined, the estimation of $m_{i}$ can be calculated by the inverse discrete Fourier transform, namely,

$$
m_{i}^{\prime}=\frac{1}{N} \sum_{n=-\lfloor N / 2\rfloor}^{\lfloor N / 2\rfloor-1} \Psi_{A N}(n) e^{-j n \varphi_{i}}
$$

Let the magnitude and phase of $\Psi_{A N}(1)$ be in the complex form, for example,

$$
\Psi_{A N}(1)=p e^{j q},
$$

where $p \in\left[0, \pi k \rho \sin \theta_{\max }\right], q \in(-\pi, \pi]$, and $\theta_{\max }$ is the maximum elevation angle. Combining (27), (28), and (29), we get the estimation of $m_{i}$, namely,

$$
\begin{array}{r}
m_{i}^{\prime}=\frac{1}{N}\left[\Psi^{\prime}\left(-\left\lfloor\frac{N}{2}\right\rfloor\right) e^{-j\left\lfloor\frac{N}{2}\right\rfloor \varphi_{i}}+\cdots+p e^{-j q} e^{-j \varphi_{i}}+\Psi^{\prime}(0)\right. \\
\left.+p e^{j q} e^{j \varphi_{i}}+\cdots+\Psi^{\prime}\left(\left\lfloor\frac{N}{2}\right\rfloor-1\right) e^{j(\lfloor N / 2\rfloor-1) \varphi_{i}}\right\rfloor .
\end{array}
$$

Furthermore, because the ambiguous numbers are integers, the real part of $m_{i}^{\prime}$ is an integer and the imaginary 


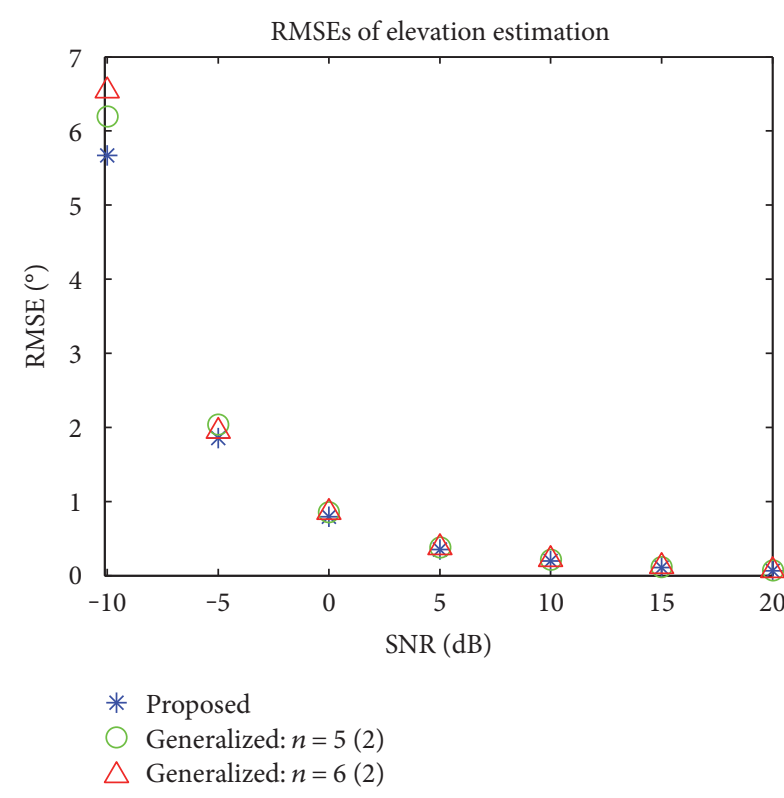

(a)

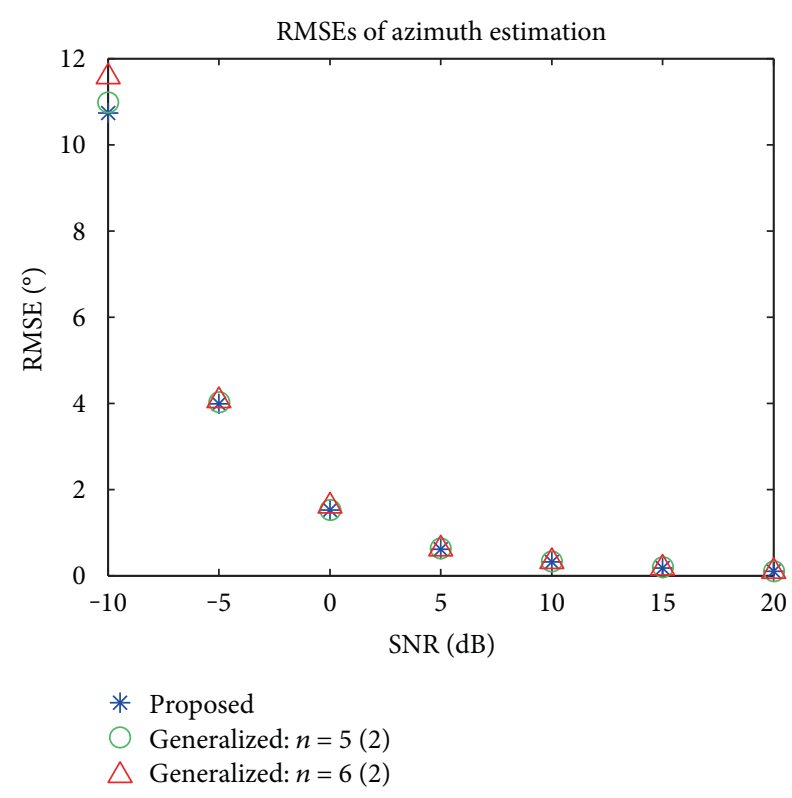

(b)

FIgURE 2: RMSEs of angle estimation. (a) Elevation angle. (b) Azimuth angle.

part of $m_{i}^{\prime}$ is zero. Considering phases noises, the following cost function approximates to zero:

$$
t=\sqrt{\sum_{i=1}^{N}\left(\left(\operatorname{real}\left(m_{i}^{\prime}\right)-\operatorname{int}\left(\operatorname{real}\left(m_{i}^{\prime}\right)\right)\right)^{2}+\operatorname{imag}\left(m_{i}^{\prime}\right)^{2}\right)},
$$

where real $(x)$ and $\operatorname{imag}(x)$ are the real and imaginary part of $x$, respectively, and int $(x)$ is the nearest integer of $x$.

Therefore, the minimum value of $t$ can be found by grid search of $p$ and $q$. The ambiguous numbers are estimated by (28), and due to noises in measured phases, $m_{i}^{\prime}$ is not an integer; then we take

$$
m_{i}=\operatorname{int}\left(m_{i}^{\prime}\right)
$$

After successful phase ambiguity resolution, unambiguous phase is calculated adopting (22), and enhanced AOA estimation accuracy can be obtained based on (12) and (13).

In ambiguity resolution, the information of every antenna is involved, and hence, the ambiguity resolution by IDFT of spectrum and integer search is robust.

\section{Simulation Results}

First, a UCA with $N=11$ antennas and a radius $\rho_{0}=\lambda / 4$ was exemplified. The incident angle of the source is $\theta_{s}=30^{\circ}$ and $\varphi_{s}=120^{\circ}$. The number of snapshots was 500. Corruption of signal samples was considered as AWGN. 1000 independent trials were run for calculating 2-D AOAs by (12) and (13). The root mean square errors (RMSEs) of elevation and azimuth angles against SNR are shown in Figure 2. The results of the generalized algorithm [2] with $n=5$ and $n=6$ were also shown for comparison. It is noticed that the
TABLE 1: Ambiguous phase, ambiguous number, and unambiguous phase.

\begin{tabular}{lccc}
\hline $\begin{array}{l}\text { Antenna } \\
\text { number, } i\end{array}$ & $\begin{array}{c}\text { Ambiguous } \\
\text { phase, } \tilde{\Phi}_{i}^{\prime}\end{array}$ & $\begin{array}{c}\text { Ambiguous } \\
\text { number, } m_{i}\end{array}$ & $\begin{array}{c}\text { Unambiguous } \\
\text { phase, } \tilde{\Phi}_{i}\end{array}$ \\
\hline 2 & 153.9 & -4 & 1593.9 \\
3 & -83.5 & -8 & 2796.5 \\
4 & 130.1 & -9 & 3370.1 \\
5 & 20.1 & -9 & 3260.1 \\
6 & -63.8 & -7 & 2456.2 \\
7 & 16.7 & -3 & 1096.7 \\
8 & 139.1 & 2 & -580.9 \\
9 & -111.5 & 6 & -2271.5 \\
10 & -100.2 & 10 & -3700.2 \\
11 & 75.5 & 13 & -4604.5 \\
12 & -170.3 & 13 & -4850.3 \\
13 & -48.2 & 12 & -4368.2 \\
14 & -25.8 & 9 & -3265.8 \\
15 & 81.5 & 5 & -1718.5 \\
\hline
\end{tabular}

proposed algorithm performs better than the generalized algorithm, because the RMSEs of the proposed method are lower than those of the generalized algorithm.

Next, a UCA with $N=15$ antennas and a radius $\rho_{0}=15 \lambda$ was exemplified for ambiguity resolution. An incident wave impinged from the angle $\theta_{s}=50^{\circ}$ and $\varphi_{s}=80^{\circ}$. Equivalent phase noises were considered as normal distribution with zero mean and variance of $\left(5^{\circ}\right)^{2}$. The phases were measured with reference to the first antenna, and the ambiguous phases are listed in Table 1. The ambiguous numbers were calculated employing (30), (31), and (32), and then the unambiguous phases were calculated using (22). At last, the elevation angle 
and azimuth angle were estimated utilizing (12) and (13), respectively. The estimated elevation angle and azimuth angle were $\theta_{s}=50.027^{\circ}$ and $\varphi_{s}=79.98^{\circ}$, respectively.

\section{Conclusions}

An analytical algorithm for accurate 2-D AOA estimation of a single source with UCAs has been proposed in this paper. 2-D AOAs are estimated in the Fourier domain. The algorithm has provided explicit and algebraic formulations for accurate azimuth and elevation angle estimations based on phase measurement by an arbitrary number of antennas no less than three. Moreover, a signal model for phase extraction has been addressed from signal samples contaminated by AWGN. Ambiguity resolution has also been realized in the Fourier domain and by integer search. Simulation results demonstrate the effectiveness and appealing performance of the proposed algorithm. However, the integer search process for ambiguity resolution can be further optimized.

\section{Conflicts of Interest}

The authors declare that they have no conflicts of interest.

\section{References}

[1] Y. Wu and H. C. So, "Simple and accurate two-dimensional angle estimation for a single source with uniform circular array," IEEE Antennas and Wireless Propagation Letters, vol. 7, pp. 78-80, 2008.

[2] B. Liao, Y. T. Wu, and S. C. Chan, "A generalized algorithm for fast two-dimensional angle estimation of a single source with uniform circular arrays," IEEE Antennas and Wireless Propagation Letters, vol. 11, pp. 984-986, 2012.

[3] Z. Ye, L. Xiang, and X. Xu, "DOA estimation with circular array via spatial averaging algorithm," IEEE Antennas and Wireless Propagation Letters, vol. 6, pp. 74-76, 2007.

[4] C. P. Mathews and M. D. Zoltowski, "Eigenstructure techniques for 2-D angle estimation with uniform circular arrays," IEEE Transactions on Signal Processing, vol. 42, no. 9, pp. 2395-2407, 1994.

[5] M. Costa, A. Richter, and V. Koivunen, "Unified array manifold decomposition based on spherical harmonics and 2-D Fourier basis," IEEE Transactions on Signal Processing, vol. 58, no. 9, pp. 4634-4645, 2010.

[6] E. Jacobs and E. W. Ralston, "Ambiguity resolution in interferometry," IEEE Transactions on Aerospace and Electronic Systems, vol. AES-17, no. 6, pp. 766-780, 1981.

[7] K. R. Sundaram, R. K. Mallik, and U. M. S. Murthy, "Modulo conversion method for estimating the direction of arrival," IEEE Transactions on Aerospace and Electronic Systems, vol. 36, no. 4, pp. 1391-1396, 2000.

[8] Z. M. Liu and F. C. Guo, "Azimuth and elevation estimation with rotating long-baseline interferometers," IEEE Transactions on Signal Processing, vol. 63, no. 9, pp. 2405-2419, 2015.

[9] X. Chen, Z. Liu, and X. Z. Wei, "Unambiguous parameter estimation of multiple near-field sources via rotating uniform circular array," IEEE Antennas and Wireless Propagation Letters, vol. 16, pp. 872-875, 2017.

[10] M. Lin, P. Liu, and J. Liu, "Rotary way to resolve ambiguity for planar array," in Proceedings of IEEE International Conference on Signal Processing, Communications and Computing (ICSPCC), pp. 170-174, Guilin, China, 5-8 August 2014.

[11] X. Chen, Z. Liu, and X. Wei, “Ambiguity resolution for phasebased 3-D source localization under fixed uniform circular array," Sensors, vol. 17, no. 5, p. 1086, 2017.

[12] S. A. Tretter, "Estimating the frequency of a noisy sinusoid by linear regression," IEEE Transactions on Information Theory, vol. 31, no. 6, pp. 832-835, 1985.

[13] S. Kay, "A fast and accurate single frequency estimator," IEEE Transactions on Acoustics, Speech, and Signal Processing, vol. 37, no. 12, pp. 1987-1990, 1989.

[14] D. Rife and R. Boorstyn, "Single tone parameter estimation from discrete-time observations," IEEE Transactions on Information Theory, vol. 20, no. 5, pp. 591-598, 1974. 


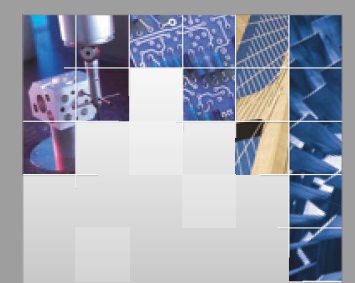

\section{Enfincering}
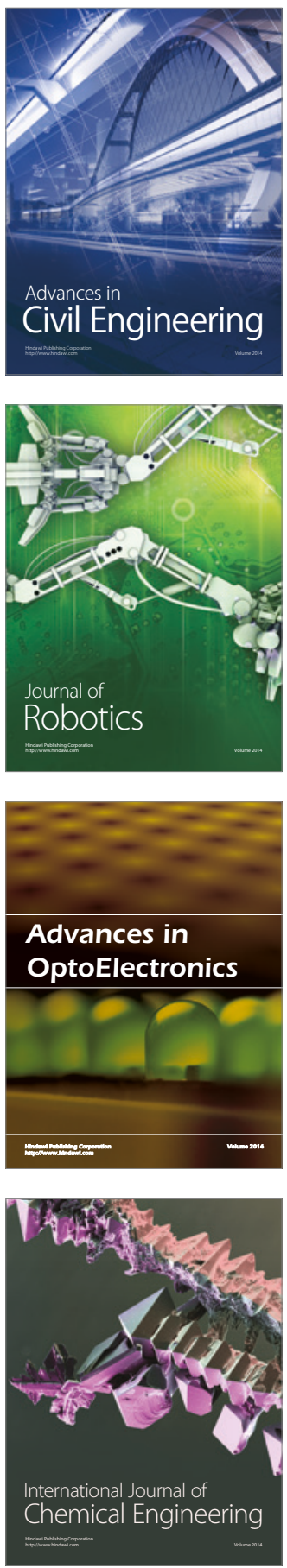

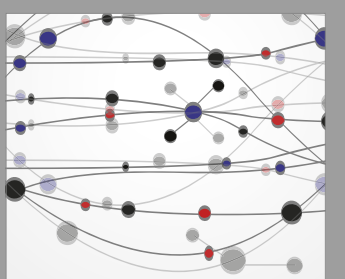

The Scientific World Journal

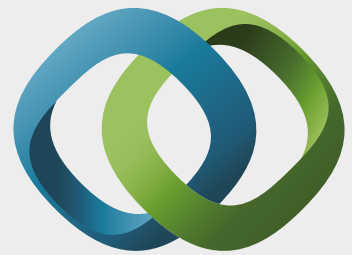

\section{Hindawi}

Submit your manuscripts at

https://www.hindawi.com
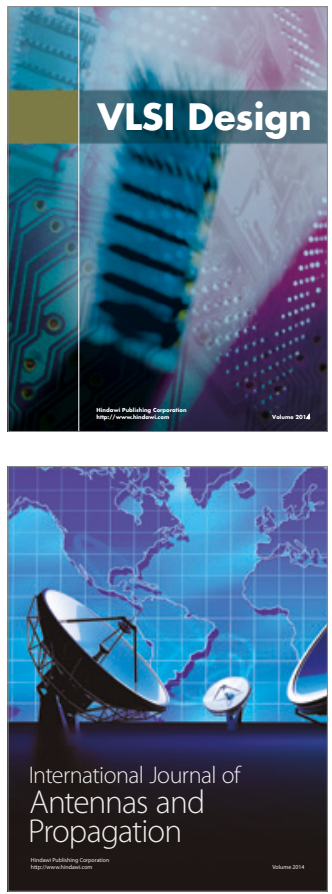

\section{Rotating}

Machinery
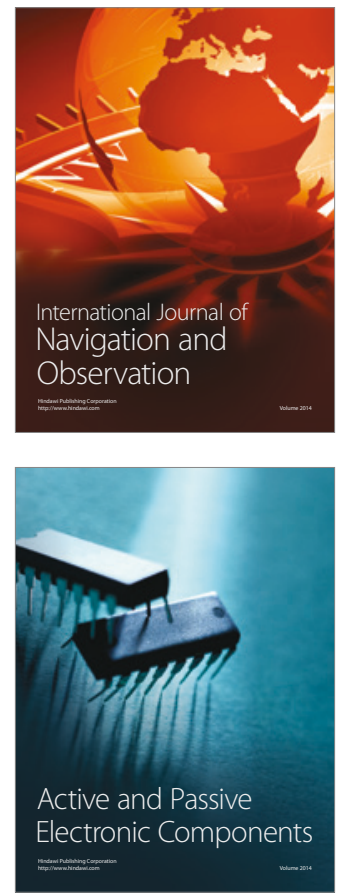
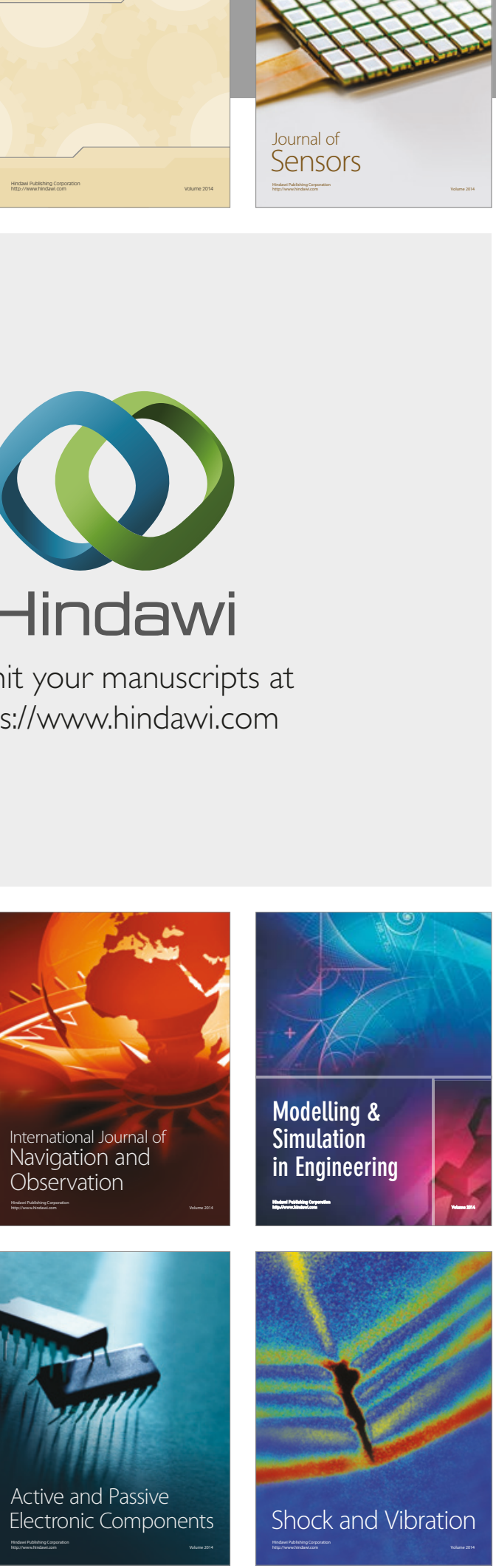
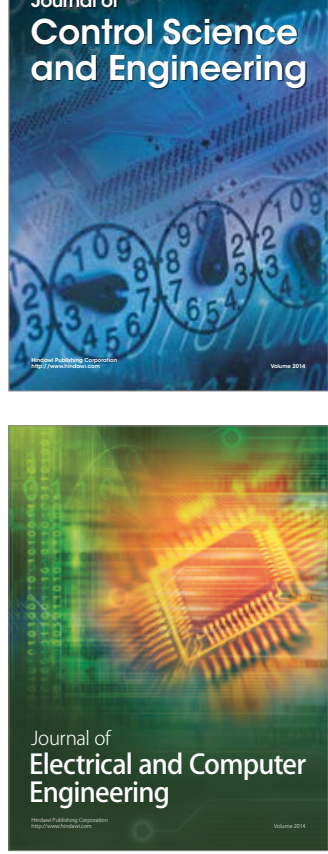

Distributed

Journal of

Control Science

and Engineering
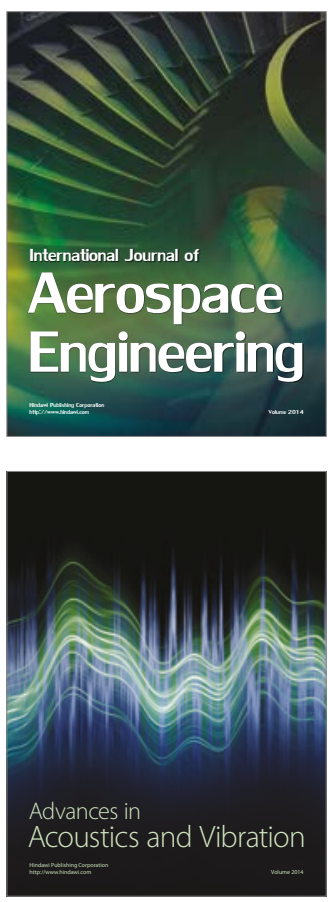

Sensor Networks 